\title{
Un caso potencial de depredación de puercoespín (Coendou sp.) por Ocelote (Leopardus pardalis) en La Reserva Natural La Mesenia-Paramillo, Jardín, Antioquia
}

\author{
Andrés Arias-Alzate \\ Camilo Botero \\ Juan Manuel Obando \\ Aburrá Natural, Medellín, Colombia \\ Carlos A. Delgado-V \\ Aburrá Natural \& Universidad CES, Medellín, Colombia
}

Aburrá Natural \& Grupo de Mastozoología Universidad de Antioquia, Medellín, Colombia; andresarias3@yahoo.es

El Ocelote o Manigordo (Leopardus pardalis), es la especie más grande del grupo Leopardus que se encuentra en América. Se distribuye desde el sur de Estados Unidos, pasando por Centro América hasta el norte de Argentina en Sur América, donde habita una gran variedad de hábitats desde sabanas, bosques húmedos, bosques montanos y bosques secos desde el nivel del mar hasta los 3000 msnm (Haines et al., 2006; Paviolo et al., 2016). Esta especie por lo general prefiere hábitat con altos porcentajes de cobertura vegetal, no obstante, se ha registrado en hábitats fragmentados con poca cobertura boscosa y en áreas periurbanas cerca de grandes ciudades (Delgado-V et al. 2016).

A lo largo de su distribución en general y dado a sus hábitos como depredador oportunista se ha registrado que la dieta del Ocelote abarca aproximadamente 50 especies de vertebrados (mamíferos, aves, reptiles y anfibios), principalmente mamíferos de tamaño pequeño ( $\leq 1 \mathrm{~kg}$ ) (e.g. Didelphis marsupialis, Oryzomys spp., Proechimys spp. Sylvilagus spp.), aunque ocasionalmente depreda vertebrados de talla mediana (1 kg $\leq 15 \mathrm{~kg}$ ) (e.g. Cuniculus paca, Dasyprocta spp. Dasypus novemcintus) (Emmons, 1987; Murray \& Gardner, 1997; Wang, 2002; Aliaga-Rosse et al., 2006; Abreu et al., 2008).

En Colombia es poco lo que se conoce sobre la ecología alimentaria de esta especie, principalmente en ecosistemas de alta montaña. Aquí documentamos evidencia del posible consumo de un puercoespín (Coendou sp.) por un Ocelote en un bosque montano al suroeste de Antioquia, Colombia. Esto representa un registro notable en cuanto a un nuevo ítem alimentario (Coendou sp.) en la dieta del Ocelote en zonas de alta montaña en Colombia.

La localidad de los registros está ubicada a $2.100 \mathrm{msnm}$ en la Cordillera Occidental, en la Reserva Natural La MeseniaParamillo, del municipio de Jardín, al suroeste de Antioquia (5³0'10.3"N; 7553'00.2"W). De acuerdo con el sistema de clasificación de Holdridge (1947), el área corresponde al bosque húmedo montano (bh-M). El registro se obtuvo a partir de una filmación directa de un individuo de Ocelote al interior de uno de los bosques del lugar.

En el video se observa un Ocelote el cual presenta tres espinas de puercoespín clavadas en su rostro: una de ellas en la zona del hocico (Figura 1A), una en la mejilla y la otra en la oreja (Figura 1B). Estas evidencias señalarían al menos las primeras evidencias y un intento de depredación de puercoespines por Leopardus pardalis para Colombia.

Estudios sobre la dieta del Ocelote han señalado una preferencia grande por especies presa de tamaño menor a $1 \mathrm{~kg}$, entre los que más se destacan son pequeños mamíferos terrestres (i.e. Didelphis marsupialis, Philander oposum, Metachirus nudicaudatus, Marmosops spp., Oryzomys spp., Proechimys spp. Sylvilagus spp., Sciurus spp. Saguinus fuscicollis, Saimiri sciureus) y algunos mamíferos voladores (Artibeus fuliginosus, Micronycteris sp.). Sin embargo, dado a las características ecológicas del Ocelote como depredador oportunista, algunos estudios han señalado el consumo de vertebrados de talla mediana, principalmente mamíferos (Emmons 1987, Wang 2002). Entre estas especies se destaca el consumo de primates (Alouatta spp. Brachyteles hypoxanthus, Sapajus nigritus, Sapajus apella), xenartros (Dasypus novemcintus, Choloepus

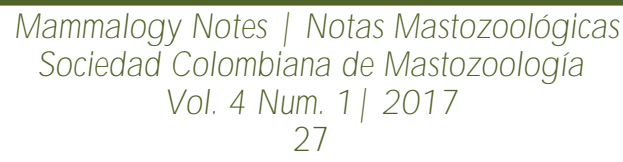


didactylus, Choloepus hoffmanni, Bradypus variegatus, Tamandua tetradactyla y T. mexicana), algunas especies de carnívoros (Nasua narica, Nasua nasua, Bassaricyon alleni, Potos flavus) y roedores (Cuniculus paca, Dasyprocta spp. Myoprocta pratti, Myocastor coypus) (Konecny 1989, Emmons 1987, Chinchilla 1997, Murray \& Gardner 1997, Wang 2002, Bianchi and Mendes 2007, Abreu et al. 2008, Delibes et al.2011, Santos et al. 2014).

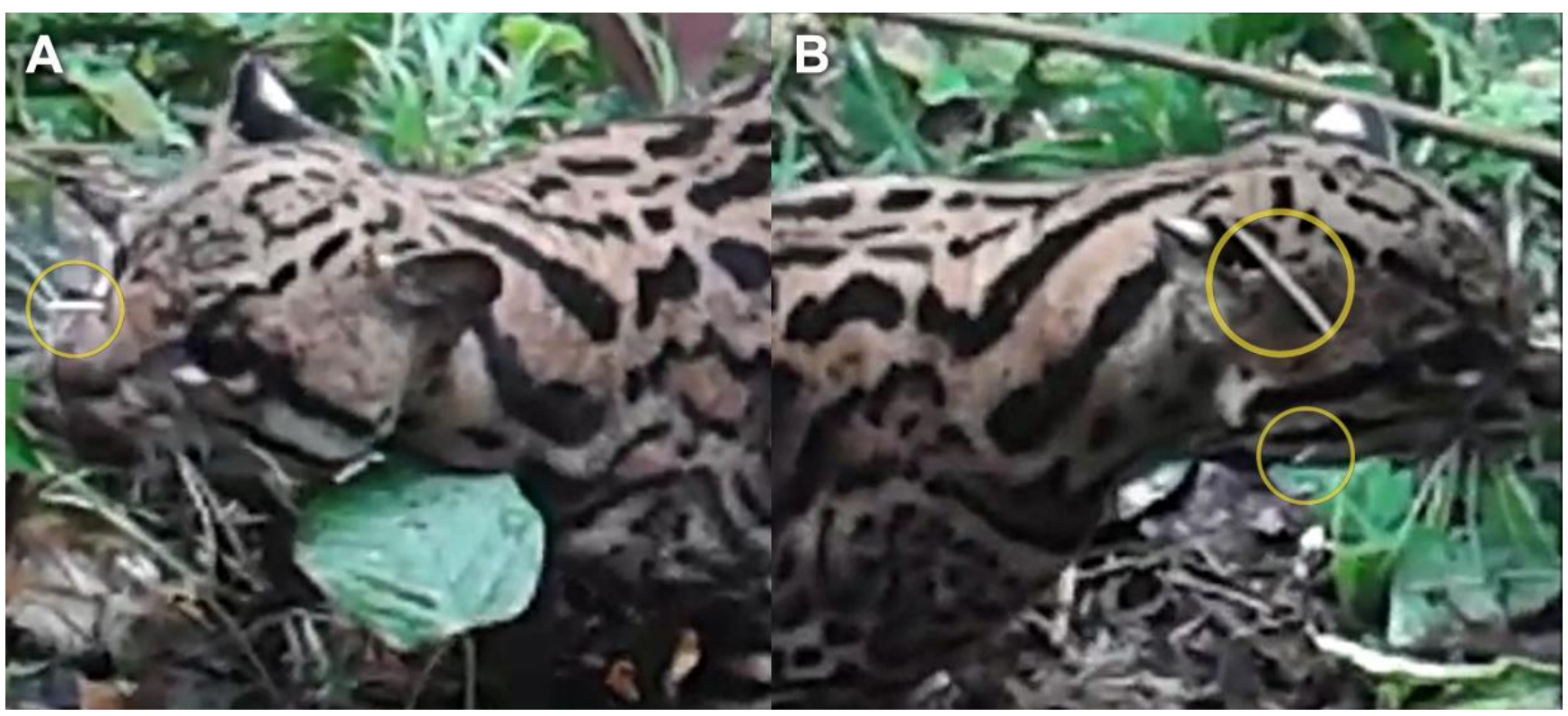

Figura 1. Ocelote (Leopardus pardalis) con espinas de puercoespín en el rostro. Documentado en la Reserva Natural La Mesenia, municipio de Jardín, suroeste de Antioquia, Colombia.

Con relación a este último grupo, aunque existen evidencias previas de depredación y del consumo de puercoespines como parte de la dieta de Leopardus pardialis, estos reportes son escasos considerando la amplia distribución de la especie y el número de especies de puercoespines ( 13 spp.) (Voss 2015), los cuales en su mayoría son simpátricos con el Ocelote. Hasta el momento solo existen registros de depredación para las especies Coendou mexicanus (Abreu et al. 2008, Murray \& Gardner 1997), Coendou prehensilis (Emmons 1987, Murray \& Gardner 1997) y Coendou rothschildi (población de Panamá ahora considerada dentro de C. quichua) (Murray \& Gardner 1997, Voss 2015). Según Voss (2015) las únicas especies de puercoespines presentes al suroriente de Antioquia (zona centro de Colombia) serían Coendou rufescens y C. quichua, por lo que este registro aquí documentado representaría una evidencia indirecta y un nuevo registro de puercoespín como potencial presa para Leopardus pardalis en Colombia. Es de resaltar que posiblemente las dos especies de puercoespines sean depredadas, por lo que esta información aquí documentada representa un nuevo registro en la dieta tanto para Colombia como en especies presa potenciales para el Ocelote.

Esperamos que este registro complemente y permita un mejor entendimiento de la dieta de Leopardus pardalis. Es importante ampliar los esfuerzos y los estudios en campo para documentar mejor este tipo de aspectos de historia natural en los felinos y otras especies de carnívoros silvestres, en especial en zonas poco estudiadas como los ecosistemas de alta montaña y hábitats circundantes a las ciudades.

\section{Agradecimientos}

La información aquí documentada hace parte de un video que es material de la Reserva Natural La Mesenia Paramillo, en el municipio de jardín, Antioquia. Agradecemos a la Reserva por permitirnos usar esta información.

\section{Referencias}

ABREU, K. C. et al. 2008. Feeding habits of ocelot (Leopardus pardalis) in Southern Brazil. Mammalian Biology 73:407-411. ALIAGA-ROSSEL, E. et al. 2006. Ocelot (Leopardus pardalis) Predation on Agouti (Dasyprocta punctata). Biotropica 38:691-694.

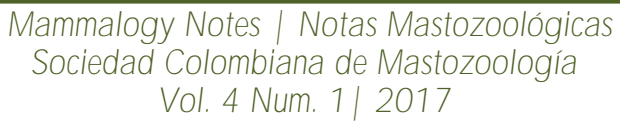


BIANCHI, R.C. \& S. L. Mendes. 2007. Ocelot (Leopardus pardalis) predation on primates in Caratinga Biological Station, Southeast Brazil. Primates 69: $1-6$.

CHINCHILLA, F. A. 1997. La dieta del jaguar (Panthera onca), el puma (Felis concolor) y el manigordo (Felis pardalis) (Carnivora: Felidae) en el Parque Nacional Corcovado, Costa Rica. 45:1223-1229.

DELIBES, M. et al. 2011. Unusual observation of an ocelot (Leopardus pardalis) eating an adult Linnaeus's two-toed sloth (Choloepus didactylus). Mammalian Biology $76: 240-241$.

DELGADO-V, C.A. et al. 2016. Naturaleza en línea: Una experiencia digital y colaborativa de biodiversidad en el Valle de Aburrá. En: Mejía et al. (ed). Naturaleza Urbana. Instituto Alexander von Humboldt. Bogotá D.C., Colombia.

EMMONS, L.H. 1987. Comparative feeding ecology of felids in a Neotropical rainforest. Behavioral Ecology and Sociobiology 20:271-283.

HAINES, A. M. et al. 2006. The importance of private lands for ocelot Leopardus pardalis conservation in the United States. Oryx 40:90.

HOLDRIDGE L.R. 1947. Determination of world plant formations from simple climatic data. Science 105 (2727): 367-368.

KONECNY, M.J., 1989. Movement patterns and food habits of four sympatric carnivore species in Belize, Central America. pp. 243-264 in Advances in Neotropical Mammalogy (Redford KH \& Eisenberg JF eds.). Sandhill Crane Press, Gainsville.

MURRAY, L., \& G. L. Gardner. 1997. Leopardus pardalis. Mammalian Species 548: 1-10.

PAVIOLO, A. et al. 2016. Leopardus pardalis. The IUCN Red List of Threatened Species 2016: e.T11509A97212355. Downloaded on 21 August 2016.

SANTOS, J. L., et al. 2014. High consumption of primates by pumas and ocelots in a remnant of the Brazilian Atlantic Forest. Brazilian Journal Of Biology 74:632-641.

VOSS, R.S. 2015. Family Erethizontidae Bonaparte, 1845. In: Patton, J.L., Pardiñas, U.F.J. and D'Elía, G. (eds), Mammals of South America, Volume 2: Rodents, pp. 786-805. The University of Chicago Press, Chicago.

WANG, E. 2002. Diets of Ocelots (Leopardus pardalis), Margays (L. wiedii), and Oncillas (L. tigrinus) in the Atlantic Rainforest in Southeast Brazil. Studies on Neotropical Fauna and Environment 37:207-212. 